\title{
ERECTA, salicylic acid, abscisic acid, and jasmonic acid modulate quantitative disease resistance of Arabidopsis thaliana to Verticillium longisporum
}

\author{
Eva Häffner ${ }^{1 *}$, Petr Karlovsky², Richard Splivallo², Anna Traczewska ${ }^{1}$ and Elke Diederichsen ${ }^{1}$
}

\begin{abstract}
Background: Verticillium longisporum is a soil-borne vascular pathogen infecting cruciferous hosts such as oilseed rape. Quantitative disease resistance (QDR) is the major control means, but its molecular basis is poorly understood so far. Quantitative trait locus (QTL) mapping was performed using a new (BurxLer) recombinant inbred line (RIL) population of Arabidopsis thaliana. Phytohormone measurements and analyses in defined mutants and near-isogenic lines (NILs) were used to identify genes and signalling pathways that underlie different resistance QTL.

Results: QTL for resistance to $V$. longisporum-induced stunting, systemic colonization by the fungus and for $V$. longisporum-induced chlorosis were identified. Stunting resistance QTL were contributed by both parents. The strongest stunting resistance QTL was shown to be identical with Erecta. A functional Erecta pathway, which was present in Bur, conferred partial resistance to V. longisporum-induced stunting. Bur showed severe stunting susceptibility in winter. Three stunting resistance QTL of Ler origin, two co-localising with wall-associated kinase-like (Wak)-genes, were detected in winter. Furthermore, Bur showed a much stronger induction of salicylic acid (SA) by $\mathrm{V}$. longisporum than Ler. Systemic colonization was controlled independently of stunting. The vec1 QTL on chromosome 2 had the strongest effect on systemic colonization. The same chromosomal region controlled the level of abscisic acid $(A B A)$ and jasmonic acid (JA) in response to $V$. longisporum: The level of ABA was higher in colonization-susceptible Ler than in colonization-resistant Bur after V. longisporum infection. JA was down-regulated in Bur after infection, but not in Ler. These differences were also demonstrated in NILs, varying only in the region containing vec1. All phytohormone responses were shown to be independent of Erecta.

Conclusions: Signalling systems with a hitherto unknown role in the QDR of A. thaliana against $V$. longisporum were identified: Erecta mediated resistance against $V$. longisporum-induced stunting. Independent of Erecta, stunting was caused in a light-dependent manner with possible participation of SA and Wakl genes. ABA and JA showed a genotype-specific response that corresponded with systemic colonization by the fungus. Understanding the biological basis of phenotypic variation in $A$. thaliana with respect to $V$. longisporum resistance will provide new approaches for implementing durable resistance in cruciferous crops.
\end{abstract}

Keywords: Arabidopsis thaliana, Verticillium longisporum, QDR, RIL, NIL, QTL, Erecta, ABA, JA, SA

\section{Background}

Quantitative disease resistance (QDR) is a complex phenomenon involving a plethora of molecular mechanisms [1,2]. It is often a sustainable form of resistance relying on multiple genes and effects which cannot easily be overcome by the pathogen's adaptation. Verticillium

\footnotetext{
*Correspondence: haeffner@zedat.fu-berlin.de

${ }^{1}$ Freie Universität Berlin, Fachbereich Biologie, Chemie, Pharmazie, Institut für Biologie, Dahlem Centre of Plant Sciences, Angewandte Genetik,

Albrecht-Thaer-Weg 6, 14195 Berlin, Germany

Full list of author information is available at the end of the article
}

longisporum is a soil-borne vascular pathogen of recent evolutionary origin with a host range centred on crucifers [3]. QDR is the only form of resistance against this pathogen described so far. $V$. longisporum causes significant and increasing yield losses on oilseed rape [4-7]. The fungus enters the host via the root and, at the onset of flowering, spreads systemically inside the xylem, thereby colonizing the whole plant $[5,8,9]$. V. longisporum induces early senescence $[6,10]$ and colonizes senescent tissue to

\section{Biomed Central}

(c) 2014 Häffner et al.; licensee BioMed Central Ltd. This is an Open Access article distributed under the terms of the Creative Commons Attribution License (http://creativecommons.org/licenses/by/2.0), which permits unrestricted use, distribution, and reproduction in any medium, provided the original work is properly credited. The Creative Commons Public Domain Dedication waiver (http://creativecommons.org/publicdomain/zero/1.0/) applies to the data made available in this article, unless otherwise stated. 
form microsclerotia that persist in the soil [11]. Since the disease is difficult to control, durable resistance in host plants is highly desirable.

Quantitative resistance against $V$. longisporum has been described for various accessions of Brassica species [12-15] (Konietzki and Diederichsen unpublished) and also for $A$. thaliana ecotypes $[8,10,16,17]$. In some cases, QTL have been identified which control resistance traits, such as fresh weight [16], chlorosis [10,16], systemic colonization, stunting, and axillary branching [8], or the area under the disease progression curve in Brassica species [15] (Konietzki and Diederichsen unpublished). However, the underlying genes and regulatory processes have rarely been identified. Secondary metabolism plays a role in resistance: A resistant line of $B$. napus produced more phenolic substances in the xylem of the hypocotyl upon infection than a susceptible line [18]. Indeed, QTL for contents of phenylpropanoid compounds co-localised with resistance QTL in B. napus [19], and it was shown that soluble phenylpropanoids played a role in $A$. thaliana defence against $V$. longisporum [20]. Rfo1 has been shown to mediate resistance against $V$. longisporum-induced fresh weight loss in A. thaliana [16], and encodes a wall-associated kinase-like (WAKL) -protein that conferred resistance against Fusarium oxysporum [21].

Recent research has revealed different processes that are involved in the host's response to $V$. longisporum on the molecular level: Ethylene signalling plays either a protective or a deleterious role, depending on the signalling components involved [16,22]. $V$. longisporum infection caused elevated levels of salicylic acid (SA) in the xylem of Brassica shoots [23]. Previous studies suggest that jasmonic acid (JA) signalling does not play a role in the host-pathogen interaction [10,23], but the JA receptor COI1 promotes the disease in a JA-independent, yet unknown way [24]. Whereas an interplay of indole glucosinolates and camalexin has been shown to be involved in early defence against $V$. longisporum in A. thaliana roots [25], reactive oxygen species played a role in defence during the later stages of the disease [26]. Furthermore, the nuclear-localised $a h l 19$ gene acted as a positive regulator of defence to $V$. longisporum and other Verticillium species in A. thaliana [27]. Several apoplastic enzymes were induced by $V$. longisporum in Brassica and possibly play a role in defence [28]. The host's reaction to the pathogen involves trans-differentiation of bundle sheath cells into functional xylem elements under the control of the vascular-related NAC domain 7 transcription factor [29].

However, little is known about how these processes relate to QDR. It is unclear whether resistance QTL represent genes within regulatory systems that have already been shown to operate in the host-pathogen interaction, or whether they constitute new components, adding to the complexity of the pathosystem. Furthermore, the role of known defence signalling pathways in natural resistance to $V$. longisporum is poorly understood so far.

The present study aims at identifying genes and signalling pathways that account for differences in QDR against $V$. longisporum in $A$. thaliana. QTL for relevant resistance traits using a new (Bur $\times$ Ler) recombinant inbred line (RIL) population have been identified. It is shown that the Erecta gene corresponded to a strong QTL mediating stunting resistance and that a functional Erecta signalling pathway mediated resistance against $V$. longisporum-induced stunting. Evidence is provided that SA, abscisic acid (ABA) and JA contents responded to $V$. longisporum in a genotype-specific way, and that changes in ABA and JA content were controlled by the same QTL that also conferred resistance to systemic colonization by the fungus.

\section{Methods}

Material

A. thaliana ecotypes Bur-0, Col-0 and Ler-0 were originally obtained from the Arabidopsis Information Service (AIS) Frankfurt [30] and maintained in-house. All other $A$. thaliana genotypes were obtained from the Nottingham Arabidopsis Stock Centre (NASC). er-105, er-108, er-111, and er-118 were included as strong erecta mutants, whilst er-116 represented a weaker erecta mutant [31,32]. agb1-1, which is defective in the $\beta$-subunit of the heteromeric G-protein, was included as a mutant of a signalling component acting downstream of Erecta [31]. The $V$. longisporum isolate '43' (V43) [33] was used for inoculation experiments.

\section{Generation of the (Bur×Ler) RIL population and near- isogenic lines (NILs)}

A total of 189 RILs were created originating from an F1 between the ecotypes Bur-0 (q) and Ler-0 ( $\hat{\jmath})$. A total of 189 F2 plants were propagated via single-seed descent to the F6 generation. All plants were grown in pots of $5 \mathrm{~cm}$ diameter in a greenhouse under long-day conditions $\left(16 \mathrm{~h}\right.$ light $/ 8 \mathrm{~h}$ dark) at $20^{\circ} \mathrm{C}$. A total of $94 \mathrm{~F} 6$ plants were genotyped and phenotyped in F7. Information about the RIL population will be submitted to the The Arabidopsis Information Resource (TAIR) database [34], and F8 seeds from bulked F7 offspring of the genotyped F6 plants will be made available through the A. thaliana stock centres Arabidopsis Biological Resource Center (ABRC) and NASC.

NILs were created by selfing RIL21, which was heterozygous for markers EH2-4 to nga361 on chromosome 2 and homozygous for all other marker loci investigated. NIL5 was selected from RIL21 offspring as homozygous for Bur alleles in the variable region, and NIL9 for Ler alleles. 


\section{Marker development and analysis}

The RILs were genotyped with 73 markers that were polymorphic between the parental ecotypes Bur and Ler. Among them were 39 simple sequence repeat (SSR) markers, 21 sequence-characterized (SCAR) markers exploiting length polymorphisms between Bur and Ler, and 12 cleaved amplified polymorphic sequence (CAPS) markers developed on the basis of single nucleotide polymorphisms (SNPs; see Additional file 1). The erecta mutation was used as a morphological marker, however, a CAPS marker (BLC2-1) has also been developed to differentiate between the Bur and the Ler allele of Erecta. Fifty-one markers have been published before $[8,34,35]$, and 22 new markers were designed to increase marker density especially in regions of major QTL (Additional file 1). Length polymorphisms between Bur and Ler were identified using the multiple SNP query tool (MSQT) database [36]. SNPs available through the seqviewer tool of TAIR $[37,38]$ were used for CAPS marker design. Webcutter 2.0 [39] was used to identify differential restriction sites at the sites of SNPs. Primers were designed on the basis of sequence information provided by TAIR.

\section{Inoculation experiments}

Inoculation experiments were performed in the greenhouse or in a growth chamber (Grobank, Mobylux, Germany) under long-day conditions ( $16 \mathrm{~h}$ light/ $8 \mathrm{~h}$ dark) on soil as described previously [8]. Thirty plants were grown in trays measuring $20 \times 30 \mathrm{~cm}$. Plants of the same treatment and genotype were arranged in batches of 15 (1/2 tray) or 30 plants (1 tray). Batch replicates were randomized. Plants flowered during the experiment and were grown until the first siliques turned yellow. Two experiments for phenotyping the RIL population were carried out with the same 94 lines (Table 1, experiments 1 and 2). Comparisons between wild type (WT) and erecta mutants were performed in two greenhouse experiments (Table 1, experiments 3 and 5), and phytohormone contents were analysed in three experiments (Table 1 , experiments 4, 5 and 6). Regarding the analysis of phytohormone contents during development in Bur and Ler (Table 1, experiment 4), plants were harvested at three different developmental stages: Stage I (earlyflowering stage) was analysed in Ler at 20 days post inoculation (dpi) and in Bur at $31 \mathrm{dpi}$. Stage II (mid- to late-flowering stage) was analysed at $27 \mathrm{dpi}$ in Ler and at 38 dpi in Bur. Stage III (onset of silique maturity) was analysed at $31 \mathrm{dpi}$ in Ler and at $48 \mathrm{dpi}$ in Bur.

\section{Phenotypic analysis}

Traits were recorded by different parameters (Table 1). Stunting resistance was measured as "performance height" (mean height inoculated $_{\text {mean height }}$ control $\times 100$ ) and "performance fresh weight" (mean $\mathrm{FW}_{\text {inoculated }}$ mean $\left.\mathrm{FW}_{\text {control }} \times 100\right)$. The performance parameters compensate for differences in plant height, and fresh weight between erecta mutants and WT. The height was measured between the hypocotyl and the apex of the longest shoot at the beginning of silique maturation when shoots were out-grown. Systemic colonization was determined in apical segments of the main shoot at the onset of silique maturity. To determine the percentage of colonized plants per replicate, one segment per inoculated plant was placed on a malt agar plate as described previously [8], and the percentage of colonized segments was calculated from batches of 15-30 plants. In order to measure the fungal DNA via $\mathrm{qPCR}, 100 \mathrm{mg}$ of shoot material was cut from segments used for plating and shock-frozen in liquid nitrogen. qPCR using Verticillium-specific primers was performed as described [40]. Chlorosis was determined by counting yellow and green rosette leaves. Three different parameters were used to quantify the trait: The number of yellow rosette leaves in inoculated plants was determined as the most direct measure of chlorosis. Furthermore, the percentage of yellow leaves relative to the total rosette leaf number was calculated to express the degree of chlorosis affecting the leaf rosette. As a third parameter, the difference in yellow leaves between inoculated and mock-inoculated plants of the same line is given, thus taking natural senescence into account. Chlorosis in both RIL experiments (experiments 1 and 2, Table 1) was assessed at $17 \mathrm{dpi}$ when the control plants still showed little or no senescence-associated chlorosis, while the $V$. longisporum-inoculated plants were showing chlorotic leaves. In order to determine the time-course of chlorosis, mock-inoculated and inoculated Ler and Bur plants were assessed every 3-4 days after inoculation in a growth chamber experiment (experiment 7, Table 1). Cotyledons were not included.

\section{Phytohormone quantification by HPLC-ESI-MS/MS}

Approximately $6 \mathrm{~cm}$ from the upper half of the stalk were sampled from 15-30 plants of the same treatment and pooled in a single sample. Material was shock-frozen in liquid nitrogen directly after harvest, subsequently lyophilized and ground to a fine powder in a ball mill. An aliquot of 50-100 mg (dry weight) was placed in $2.0 \mathrm{ml}$ tubes and used for phytohormone extraction according to a modified protocol [23]. One $\mathrm{ml}$ extraction solvent (20\% acetone, $79 \% \mathrm{H}_{2} \mathrm{O}$ and $1 \% \mathrm{CH}_{3} \mathrm{COOH}$, and $2 \mathrm{ng}$ of the deuteriated internal standard D6-salicylic acid, Sigma-Aldrich Co.) was added to each sample. Extraction was performed for $45 \mathrm{~min}$ at $4^{\circ} \mathrm{C}$ on a rotary shaker $(160 \mathrm{rpm})$. Subsequently, each sample was spiked with $1.0 \mathrm{ml}$ diethyl ether (DEE), shortly vortexed, and shaken $\left(160 \mathrm{rpm}, 4^{\circ} \mathrm{C}\right)$ for an additional $30 \mathrm{~min}$. The sample was centrifuged at $8500 \mathrm{~g}$ for $5 \mathrm{~min}$. The upper DEE phase was transferred to a new 
Table 1 Overview of inoculation experiments (exp.)

\begin{tabular}{|c|c|c|c|c|c|c|}
\hline $\begin{array}{l}\text { Exp. } \\
\text { no. }\end{array}$ & Genotypes & Traits & $\begin{array}{l}\text { Plant replicates } \\
\text { control/V43 }\end{array}$ & $\begin{array}{l}\text { Batch replicates } \\
\text { controlcV43 }\end{array}$ & Date & Environment \\
\hline 1 & Bur, Ler, 94 RILs & $\begin{array}{l}\text { Systemic colonization }{ }^{1)} \text {, stunting }{ }^{2)} \\
\text { chlorosis }^{3)} \text {, development time }\end{array}$ & $\begin{array}{l}15 / 30 \text { for RILs, } 90 / 90 \\
\text { for Bur and Ler }\end{array}$ & $\begin{array}{l}1 / 1 \text { for RILs, } 6 / 6 \\
\text { for Bur and Ler }\end{array}$ & $\begin{array}{l}01 / 2009- \\
03 / 2009\end{array}$ & Greenhouse \\
\hline 2 & Bur, Ler, 94 RILs & $\begin{array}{l}\text { Systemic colonization, stunting, } \\
\text { chlorosis development time }\end{array}$ & $\begin{array}{l}15 / 30 \text { for RILs, } 90 / 90 \\
\text { for Bur and Ler }\end{array}$ & $\begin{array}{l}\text { 1/1for RILs, } 6 / 6 \text { for } \\
\text { Bur and Ler }\end{array}$ & $\begin{array}{l}04 / 2009- \\
06 / 2009\end{array}$ & Greenhouse \\
\hline 3 & $\begin{array}{l}\text { La-0, Ler, Col-0, er-105, er-108, } \\
\text { er-111, er-116, er-118, agb1-1 }\end{array}$ & $\begin{array}{l}\text { Systemic colonization, stunting, } \\
\text { chlorosis }\end{array}$ & $30 / 300$ & $1 / 10$ & $\begin{array}{l}01 / 2010- \\
03 / 2010\end{array}$ & Greenhouse \\
\hline 4 & Bur, Ler & $\begin{array}{l}\text { Phytohormone contents in different } \\
\text { developmental stages }\end{array}$ & $90-120 / 90-120$ & $6-8 / 6-8$ & $\begin{array}{l}12 / 2009- \\
02 / 2010\end{array}$ & Greenhouse \\
\hline 5 & La-0, Ler, Col-0, er-105, Bur & Phytohormone contents & $180 / 180$ & $6 / 6-7$ & $\begin{array}{l}01 / 2011- \\
03 / 2011\end{array}$ & Greenhouse \\
\hline 6 & Bur, Ler, NIL5, NIL9 & Phytohormone contents & $90 / 90$ & $6 / 6-7$ & $\begin{array}{l}09 / 2012- \\
11 / 2012\end{array}$ & Greenhouse \\
\hline 7 & Bur, Ler & Chlorosis & $60 / 60$ & $2 / 2$ & $\begin{array}{l}08 / 2008- \\
10 / 2008\end{array}$ & $\begin{array}{l}\text { Growth } \\
\text { chamber }\end{array}$ \\
\hline
\end{tabular}

Assessed parameters per trait for phenotyping: ${ }^{1)}$ a) Percentage of colonized shoot segments, b) fungal DNA content [pg/mg fresh weight], ${ }^{2)}$ a) performance height, b) performance fresh weight, ${ }^{3)}$ a) number of yellow leaves, b) percentage of yellow leaves, c) difference in number of yellow leaves between inoculated and mock-inoculated plants, ${ }^{4)}$ days to flowering in mock-inoculated plants. Plant replicates $=$ number of individual plants; batch replicates $=$ number of batches of 15 or 30 plants growing in a tray.

$1.5 \mathrm{ml}$ tube, and the remaining aqueous phase was reextracted with $1.0 \mathrm{ml}$ DEE exactly as in the previous step. Both DEE fractions were pooled and dried under vacuum at $30^{\circ} \mathrm{C}$, re-dissolved in $200 \mu \mathrm{lPLC}$ (high-performance liquid chromatography) solvent $\left(1: 1 \mathrm{H}_{2} \mathrm{O}: \mathrm{MeOH}\right.$ containing $7 \mathrm{mM} \mathrm{CH}_{3} \mathrm{COOH}$ ), centrifuged once more at $8500 \mathrm{~g}$ for $5 \mathrm{~min}$ and transferred $(180 \mu \mathrm{l})$ to an HPLC conical vial (200 $\mu$ linternal volume, WICOM Germany).

Extracted samples $(10 \mu \mathrm{l})$ were injected into the HPLC and eluted at $40^{\circ} \mathrm{C}$ at a flow rate of $0.2 \mathrm{ml} / \mathrm{min}^{-1}$. Chromatography was performed on a Kinetex ${ }^{\circ}$ C18 $(100 \mathrm{~mm} \times$ $2.10 \mathrm{~mm}$ with $2.6 \mu \mathrm{m}$ particle size) column equipped with a C18 guard column purchased from Phenomex Inc. (Aschaffenburg, Germany). The following programme was used for elution: $80 \%$ solvent A (water:acetonitrile 95:5) containing $7 \mathrm{mM} \mathrm{CH}_{3} \mathrm{COOH}$ and $20 \%$ solvent $\mathrm{B}$ (methanol containing $7 \mathrm{mM}$ acetic acid) for $40 \mathrm{~s}$; ramp to $98 \% \mathrm{~B}$ in $50 \mathrm{~s}$; hold for 2 min $20 \mathrm{~s}$ followed by re-equilibration to $20 \%$ B.

Phytohormones were detected in multiple reaction monitoring mode (MRM) in a triple quadrupole mass spectrometer (LC12000) equipped with an electrospray interface using settings described in [41]. The following mass transitions (collision energy: CE) were used: SA 136.8/93.0 (CE 14.5 eV); d6-SA 140.9/97.0 (18.5 eV), JA 208.9/59.0 (9.5 eV), and ABA 262.8/153.0 (8.0 eV). A calibration curve of the ratio of peak areas of the unlabelled standard to the peak area of the deuterium-labelled standard was used for the quantification of SA. Other phytohormones were quantified with an external calibration curve obtained with pure standard.

\section{Map construction and QTL analysis}

Linkage groups were determined and allele frequencies were tested for segregation distortion with JoinMap [42].
Map construction and QTL analysis was performed with MapManager QTX 20b [43] using the Haldane mapping function. Simple interval mapping was performed scanning the genome in $1 \mathrm{cM}$-steps. MapManager QTX gives the LRS (likelihood ratio statistic) value to assess the probability of a false positive, where LRS $=4.6 \times$ LOD (likelihood of odds) [44]. LRS significance threshold values for the $37 \%$ (suggestive), 95\% (significant) and 99.9\% (highly significant) genome-wide confidence levels were determined by permutation tests with 10,000 permutations. Confidence intervals for QTL were determined by bootstrap tests which calculate the QTL position for multiple resampled datasets of the original dataset. Epistatic interactions were searched using the "interaction" function of MapManager QTX $20 \mathrm{~b}$, testing pairs of markers for a possible interaction component. The confidence criterion for the total effect of a marker pair was set to $p=10^{-5}$. MapQTL 6 [45] was also used for mapping to include covariates and cofactors in the QTL analyses. Cofactors were initially specified according to peak positions in interval mapping and selected by backwards elimination using the "Automatic cofactor selection" tool of MapQTL" 6 . Maps were visualized using MapChart [46].

\section{Statistics}

All inoculation experiments were performed with batches of at least 15 plants per genotype and treatment. Most inoculation experiments were performed on 6 to 10 batch replicates. Some parameters, such as phytohormone contents, the performance parameters or the percentage of colonized shoot segments, were calculated on a batch basis, whereas some of the chlorosis parameters were calculated on a single-plant basis. Only one batch of 15-30 plants was inoculated per genotype to allow testing of 96 genotypes at 
the same time in the RIL inoculation experiments. The whole experiment was repeated to confirm the results. See Table 1 for an overview of single-plant and batch replicates. All statistical analyses, as indicated in the Results section, were performed with SPSS 20 [47].

\section{Results}

QTL controlling resistance traits against $V$. longisporum in the (Bur×Ler) RIL population

The parental ecotypes Bur and Ler differed in important resistance traits against $V$. longisporum (Figure 1). Ler was susceptible to systemic colonization by the pathogen, whereas Bur showed a high degree of resistance (Figure 1a). Bur, however, was more susceptible to $V$. longisporum-induced stunting than Ler. This phenotype was suppressed under high-light conditions in the greenhouse during summer (Figure 1c, d). V. longisporum promoted chlorosis in both Ler and Bur compared to mock-inoculated controls. Both natural and $V$. longisporum-induced chlorosis occurred much earlier in the early-flowering ecotype Ler than in the late-flowering ecotype Bur (Figure 1e).

QTL controlling $V$. longisporum resistance traits were mapped in a new (Bur $\times$ Ler) RIL population. A genetic map was calculated and a physical map was produced using known positions of each marker on the Arabidopsis Genome Initiative (AGI) reference map [48] (Additional file 2 ). In the genetic map, chromosomes were supported as linkage groups with LOD scores ranging from 4 (chromosome 1) to 10 (chromosome 4). Genetic marker order on each chromosome was the same as for the physical map. The complete map size was $407.3 \mathrm{cM}$, with an average marker spacing of $5.7 \mathrm{cM}$ and the largest distance between two markers being $17.6 \mathrm{cM}$. The proportion of heterozygous markers in F6 was 3.65\%, which agreed well with a predicted value of $3.125 \%$.

QTL were detected for all three resistance traits (Figure 2, Additional file 3). QTL controlling Verticillium colonization (vec) were detected on chromosomes 2 and 4, QTL for stunting resistance (stre and r-stre) on chromosomes 1, 2 and 4, and QTL for resistance against $V$. longisporum-induced chlorosis $(r$-chl) also on chromosomes 1,2 and 4. QTL were named according to the nomenclature used previously [8]. QTL exclusively discovered in the RIL population were prefixed with an "r-", indicating their origin from RIL mapping. All parameters characterizing the traits showed either a normal or a multi-modal frequency distribution, both of which can occur in traits controlled by several QTL (Additional file 4).

All vec QTL controlling systemic colonization consisted of arrays containing several LRS peaks depending on the parameter and the inoculation experiment (Figure 2, Additional file 3). The map positions of the peaks were reproducible for both parameters (plating assay and $\mathrm{qPCR}$ ) and in both inoculation experiments (Additional file
3). vec1 explained up to $19.3 \%$ of the trait variance, depending on the parameter and the experiment, vec2 up to $15 \%$ and vec3 up to $17.3 \%$ (Additional file 3). The vec1 QTL could be confirmed in NILs that differed in a $\sim 3$ megabase-segment on chromosome 2 comprising vec1. NIL9 with Ler alleles in the variable region was more susceptible to systemic colonization than NIL5 with Bur alleles in the respective region (Figure 1a). All vec alleles increasing the degree of colonization were of Ler origin. Thus, colonization resistance was entirely conferred by the Bur alleles of the respective QTL. The co-localisation of colonization and developmental QTL on chromosome 4 (Additional file 3) suggested an impact of development on systemic colonization, although all assessments were made at defined developmental stages. Development parameters were used as covariates in interval mapping or multiple QTL mapping (MQM) with MapQTL. Using the developmental parameters as a covariate, all vec QTL on chromosome 4 vanished, and vec1 on chromosome 2 remained as the only chromosomal region showing a significant effect on this resistance trait. Co-factor selection in the QTL region on chromosome 2 and application of the selected co-factors in MQM confined the QTL region controlling the degree of systemic colonization (both parameters) to a fragment between markers EH2-6, BLC2-2 and erecta, spanning approximately $1.6 \mathrm{cM}$.

QTL for stunting resistance could only be detected with the parameter "performance height" and not with "performance fresh weight". Both QTL on chromosome 2 were detected in both inoculation experiments; the QTL on chromosomes 1 and 4 were only detected in experiment 1 , which was performed in the winter. stre 2 co-localised with marker BLC1-26, which is located in the Wakl9 gene, and explained up to $22.9 \%$ of the trait variance. stre 1, explaining $16.8 \%$ variance, was also located in the vicinity of a Wakl gene (Wakl22/rfo1). $r$-stre1 near marker erecta on chromosome 2 explained approximately $30 \%$ of the trait variance in experiment 1 (Figure 2, Additional file 3). The stunting resistance alleles of the QTL on chromosome 2 were of Bur origin, whereas the stunting resistance QTL on chromosomes 1 and 4 were contributed by Ler. The fact that both parents contributed stunting resistance QTL explains the strong transgressive segregation of the trait (Additional file 4). As for the degree of systemic colonization, the development apparently had an impact on the degree of stunting. Including development traits as a covariate in mapping reduced the number of QTL controlling this trait by eliminating the QTL r-stre3 on chromosome 4, which had been detected in experiment 1 .

Significant QTL for chlorosis parameters $(r$-chl) were only detected in experiment 1 (Figure 2, Additional file 3). $r$-chl1 on chromosome 4 was the strongest QTL explaining up to $29.3 \%$ of the trait variance. $r$-chl 2 on chromosome 2 

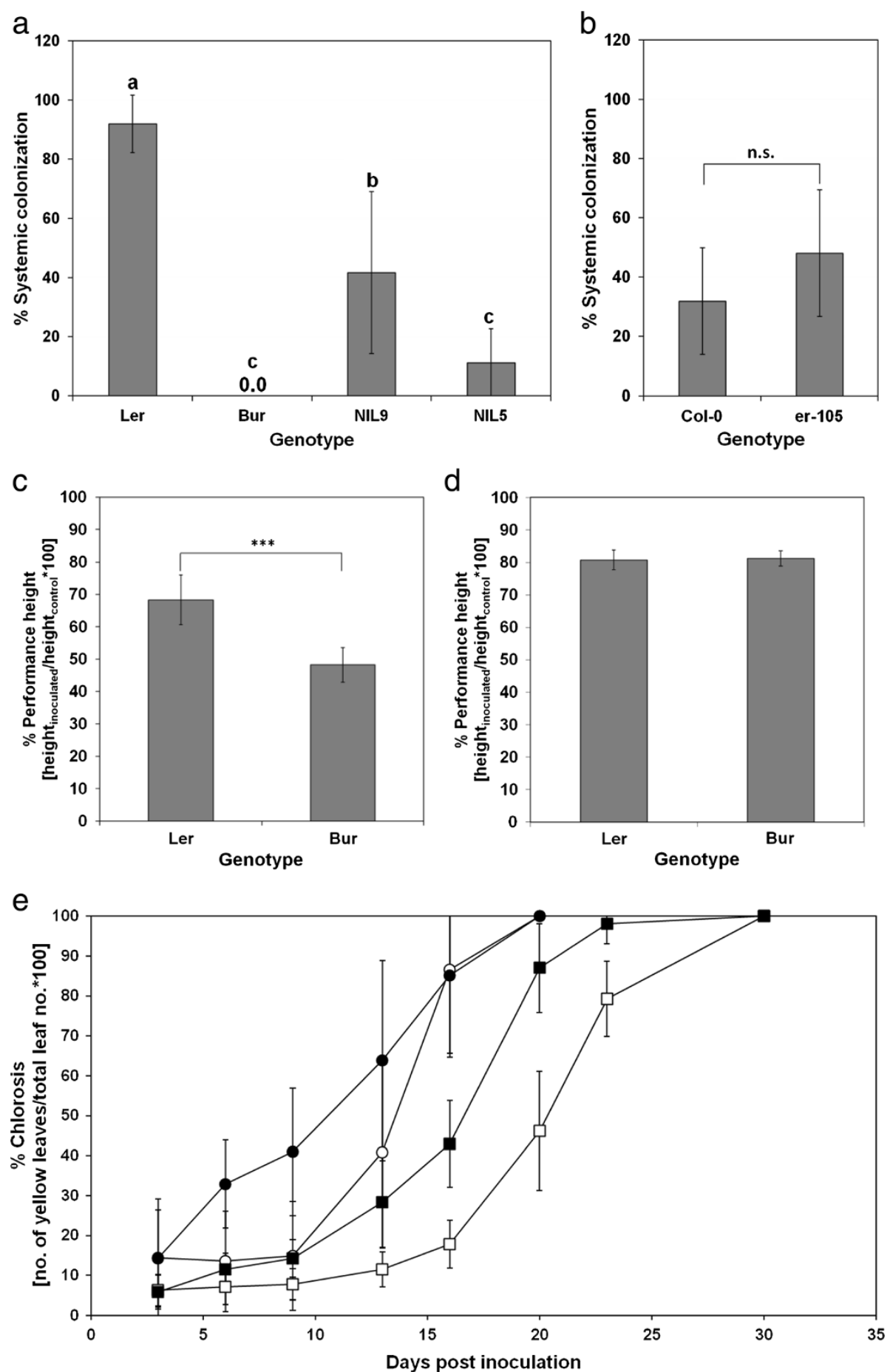

Figure 1 Phenotypic characterization of different $\boldsymbol{A}$. thaliana genotypes. a,b) Systemic colonization of a) Ler, Bur and two NILs differing in the vec1 region (experiment 6; one-way ANOVA and post-hoc Tukey test, $p<0.05, n=6-7$ ), and b) two genotypes differing only in Erecta function (experiment 5 , $t$-test, $p<0.05, n=6$ ). c,d) Resistance to $\mathrm{V}$. longisporum-induced stunting expressed as performance height in $\mathbf{c}$ ) an experiment performed in winter (experiment $1, t$-test, $p<0.05, n=6$ ) and $\mathbf{d}$ ) an experiment performed in spring (experiment $2, t$-test, $p<0.05, n=6$ ). e) Proportion of chlorotic rosette leaves in Bur (squares) and Ler (circles) after $V$. longisporum-inoculation (closed symbols) and mock-inoculation (open symbols) during the course of a growth chamber experiment. Vertical bars denote standard deviations. Ecotypes and treatments differed significantly from $6 \mathrm{dpi}$ on $(p<0.05$; t-test; $n=41$ for Ler mock, $\mathrm{n}=38$ for Ler V. longisporum, $\mathrm{n}=60$ for Bur mock, $\mathrm{n}=58$ for Bur $\mathrm{V}$. longisporum). 


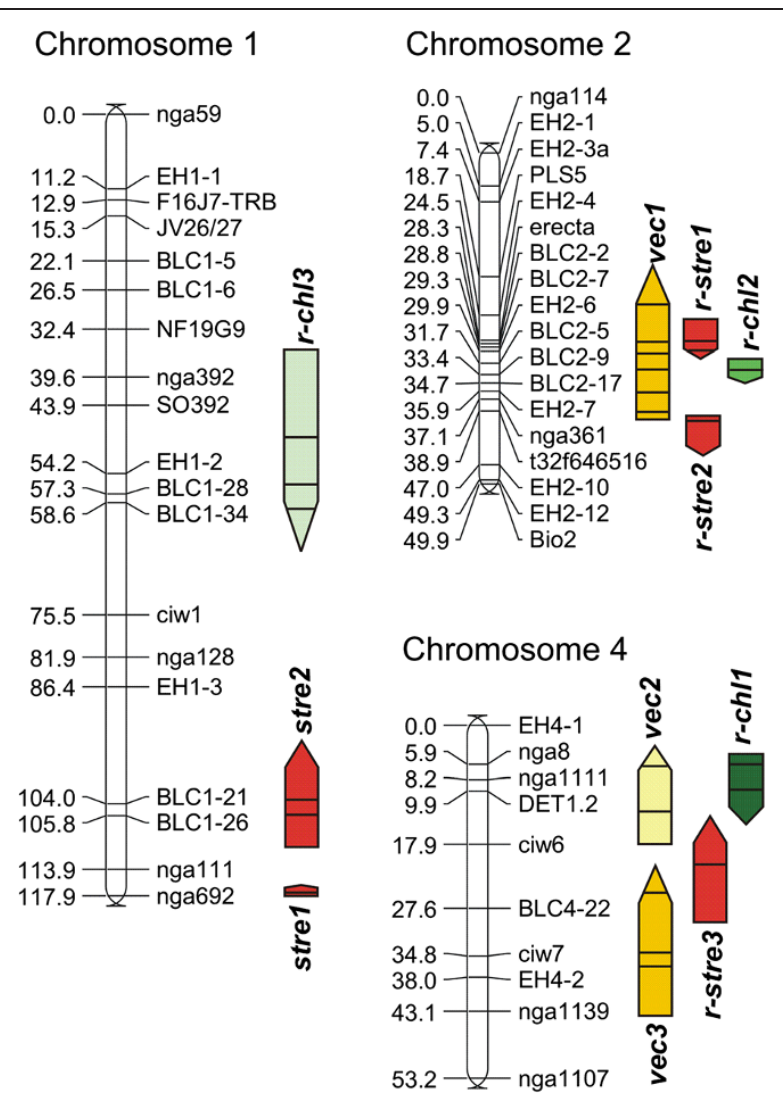

Figure 2 QTL for $V$. longisporum-related traits in the $A$. thaliana (BurXLer) RIL population. White bars represent chromosomes with marker positions indicated in CM. Coloured bars delimit the confidence intervals of QTL as follows: Light yellow: Systemic colonization, \% colonized shoot segments, exp. 1. Dark yellow: Systemic colonization, \% colonized shoot segments, exp. 2. Red: Stunting resistance, performance height, exp. 1. Dark green: Chlorosis, number of yellow leaves, exp. 1. Medium green: Chlorosis, $\%$ yellow leaves, exp. 1. Light green: Chlorosis, difference in yellow leaves between mock-inoculated and $V$. longisporum-inoculated plants, exp. 1. Black lines within the bars stand for LRS peak positions. Upward arrows indicate that the paternal ecotype (Ler) was the origin of the increasing allele; downward arrows stand for Bur as the source of the increasing allele. QTL from the most representative dataset are displayed in Figure 1. QTL names are written next to the bars. For reproducibility and further QTL information, see Additional file 3.

explained $11.7 \%$ and $r$-chl3 on chromosome 1 up to $14.8 \%$. Surprisingly, all alleles conferring resistance to chlorosis were of Ler origin. Two QTL accelerating flowering time of Ler origin were found in the same region ( $d t 1$ and $d t 2$, see Additional file 3). In general, a later onset of flowering is expected to correlate with a later onset of senescence. Furthermore, using development traits as a covariate did not affect $r$-chl1 on chromosome 4 . Both facts are strong evidence that QTL have been mapped that were specific for $V$. longisporum-induced chlorosis and not QTL controlling development.
Epistatic interactions between marker loci could not be reproduced between the tests. Regarding the colonization data determined by the plating assay in experiment 1 , a significant interaction between marker loci BLC2-7 on chromosome 2 and nga1111 on chromosome 4 was detected. Both loci lie within the confidence intervals of vec1 and $v e c 2$, respectively. In summary, it could be shown that QTL from both parents controlled different resistance traits against $V$. longisporum in the (Bur $\times$ Ler) RIL population.

\section{V. longisporum-induced stunting was controlled by ERECTA signalling}

The strong stunting resistance QTL $r$-stre1 co-localised with the morphological marker erecta. Erecta encodes for a receptor-like kinase with many functions in plant development and also in QDR [49]. The function of this protein in Ler is disrupted [32], while Bur contains a functional Erecta allele. Inoculation experiments were performed on erecta mutants and their respective WT ecotypes having a functional Erecta allele to investigate whether a functional Erecta pathway increases resistance. Ler was compared with the La-0 ecotype. Col-0 was compared with erecta mutants in the Col-0 background. The agb1-1 mutant in the Col-0 background that is defective in the $\beta$-subunit of heteromeric G-protein was included representing another component of the Erecta signalling pathway [31]. All erecta mutants and the agb1-1 mutant were significantly more stunted under $V$. longisporum challenge than their respective WT lines: The parameters "performance height" and "performance fresh weight" were decreased in all mutants compared to WT (Figure 3a, b). The weaker er-116 mutant did not perform better than the strong mutants. The developmental phenotype of er-116, however, was clearly attenuated: The mean height of the controls was $22.3 \mathrm{~cm}$ compared to $16.5 \mathrm{~cm}$ in the strong erecta mutants. A functional Erecta did not contribute significantly to colonization resistance in any of the two backgrounds (Figure 1b, Additional file 5), despite the strong colonization QTL vec1 that was localized near Erecta. Because one QTL for chlorosis ( $r$-chl2) was localized in the Erecta region, it was investigated whether loss of Erecta function influences chlorosis. The level of chlorosis in Col-0 was higher than in the erecta mutants with Col-0 background (Figure 3c), suggesting a chlorosis-promoting effect of Erecta. However, the effect of Erecta was opposite in La-0/Ler: La-0 suffered less chlorosis than Ler (Figure 3c). Thus, a background-specific effect of Erecta on chlorosis must be assumed.

It was demonstrated that Erecta underlay stunting resistance QTL $r$-stre1 and that a functional ERECTA signalling pathway mediated stunting resistance in A. thaliana. 

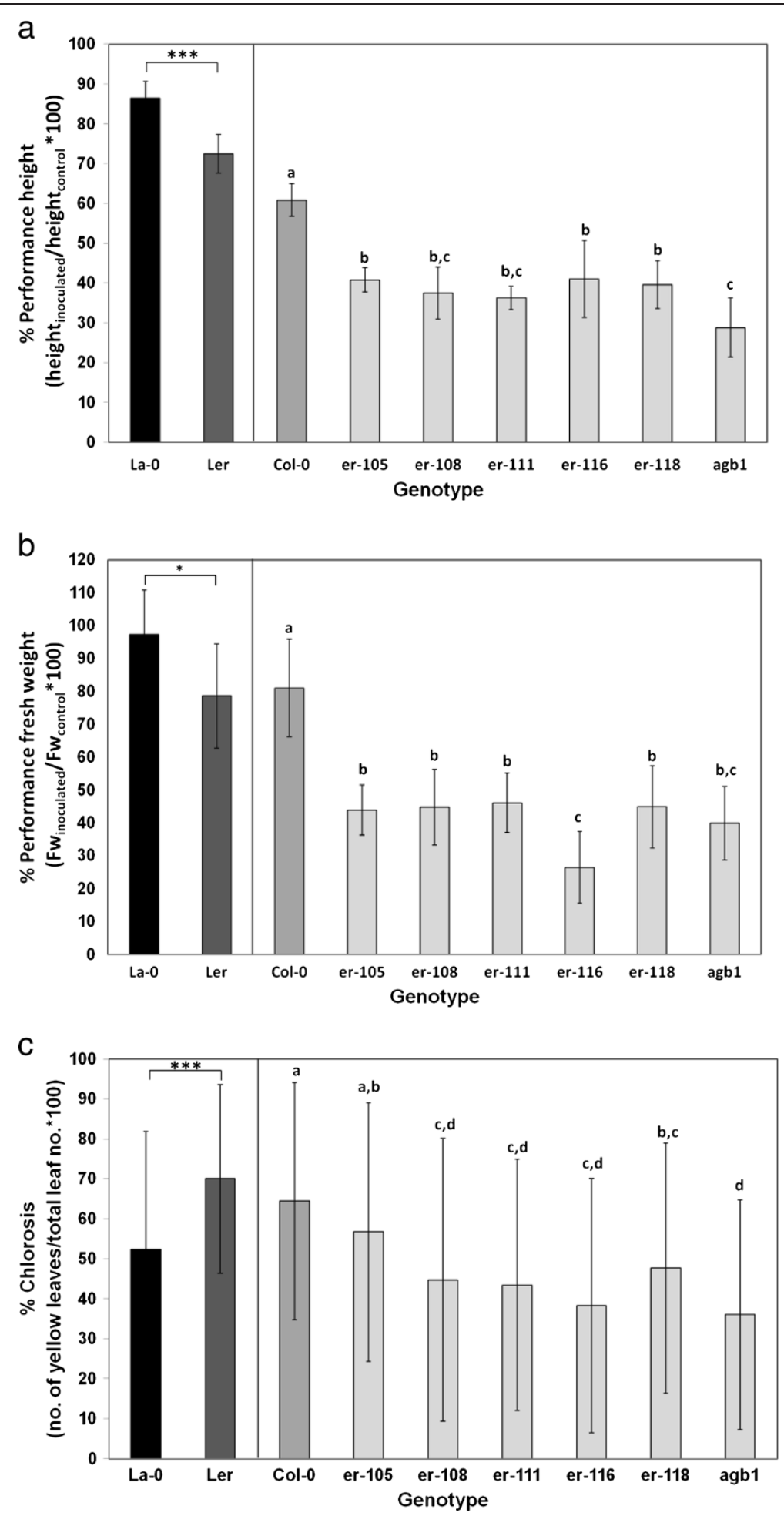

Figure 3 Reaction of Erecta signalling mutants and corresponding WT lines to $\boldsymbol{V}$. longisporum. La-0 is the corresponding WT for Ler, Col-0 for er-105, er-108, er-116, er-118, and agb1-1. Different WT/mutant combinations are separated by a vertical bar. Recorded parameters are a) performance height, b) performance fresh weight and c) \% chlorotic leaves in inoculated plants. $N=10$ for $\mathbf{a}, \mathbf{b}$ ), $\mathrm{N}$ between 91 and 260 for $\mathbf{c}$ ). Significance of La-0/Ler differences was tested by t-tests; differences between lines with Col-0 background by one-way ANOVA and subsequent multiple comparisons (Tukey test). Means marked with different letters differed significantly at $p<0.05$. Vertical bars denote standard deviations. 


\section{SA and low light were associated with $V$. longisporum- induced stunting}

SA content increased in A. thaliana stalks after infection with $V$. longisporum in both Bur and Ler (Figure 4). SA levels were strongly increased in Bur at early- to midflowering stages, while SA showed a mild but significant increase in Ler with the advance of flowering (Figure 4). SA hyperinduction was neither associated with vec1 nor with Erecta, as could be shown in NILs and erecta mutants (Additional files 6 and 7). Furthermore, Bur showed high susceptibility to $V$. longisporum-induced stunting in winter experiments (Figure 1c). This seasondependent susceptibility could be attributed to the Bur alleles of QTL stre1, stre2 and r-stre3, the first two colocalising with $\mathrm{Wakl}$ genes. Greenhouse experiments were always performed in long-day conditions with additional illumination at temperatures between 20 and $25^{\circ} \mathrm{C}$. However, the intensity, dosage and spectral composition of ambient light were major factors differing between spring/summer and winter experiments: Quantum flux densities of artificial illumination alone ranged between 80 and $100 \mu \mathrm{mol}^{*} \mathrm{~s}^{-1 *} \mathrm{~m}^{-2}$, whereas additional natural daylight provided between 200 and $400 \mu \mathrm{mol}^{*} \mathrm{~s}^{-1 *} \mathrm{~m}^{-2}$.

It is hypothesized that SA induction and low light caused $V$. longisporum-induced stunting mediated by the seasonally influenced QTL stre1, stre2 and $r$-stre3 (see Discussion).

\section{$A B A$ and $J A$ response to $V$. longisporum infection was controlled by vec1}

Bur and Ler differed in their ABA and JA responses to $V$. longisporum: ABA contents were much higher in Ler than in Bur upon infection (Figures 5 and 6). Changes of JA levels were opposite in Ler and Bur at the maturity stage: the JA level in Ler stayed the same or increased, whilst the JA levels in Bur dropped in infected plants compared to mock-inoculated plants (Figures 5 and 6). Analysis of ABA and JA content in NILs at the maturity stage showed that this difference was associated with the genomic region containing vec1 and Erecta on chromosome 2 (Figure 6). NIL9, which was homozygous for Ler alleles in the variable region, showed strong ABA induction and no JA decrease - a reaction that was similar to the one in Ler. NIL5 with Bur alleles in the variable region showed a reaction of both phytohormones that resembled Bur: ABA increased less strongly and JA decreased in infected plants (Figure 6). The characteristic phytohormone response patterns corresponded to high systemic colonization in Ler and NIL9 and low colonization in Bur and NIL5 (Figure 1a). No effect of Erecta on ABA and JA levels in $V$. longisporum-infected plants was found when erecta/WT comparisons were made for ABA and JA contents (Additional file 7). ABA and JA signalling pathways are candidates for the control of systemic colonization by $V$. longisporum in A. thaliana.

\section{Discussion}

Considerable progress has been made in understanding the molecular interactions of $V$. longisporum with its cruciferous hosts at a molecular level during recent years. Many genes and pathways have been shown to be involved, revealing an enormous complexity. The present study has been designed to further disentangle the complex network and to identify new components of genetic variation contributing to quantitative resistance to $V$. longisporum.

Our results corroborate the view that different resistance traits are controlled by different pathways and can be inherited independently. A plant showing a high degree of colonization resistance may still be susceptible to stunting. No single master mechanism controlled QDR in A. thaliana. Nevertheless, a higher resistance level can be achieved by combining a few well-defined QTL. Some components of the physiological basis of resistance were elucidated.

Phenotypic variation for colonization resistance and stunting resistance was assigned to specific QTL that have been partly detected before, but were mapped with much higher resolution in the RIL population. vec1, vec2 and vec 3 were found to control systemic colonization by $V$. longisporum in an F2/F3 population originating from the same parents [8] and could be reproduced in the present study. RIL mapping revealed a high complexity of the vec QTL. Multiple, reproducible LRS peak positions suggest complex loci in which more than one gene contributed to the effect. The genes underlying the vec QTL are still unknown, but recent results strongly suggest that genes involved in the phenylpropanoid pathway underlie vec3 [20]: The genes cad5, cad8 and ugt84a3, encoding for cinnamyl alcohol dehydrogenases and an UDP-glycosyl-transferase, are all located on chromosome 4 close to vec3. Soluble phenylpropanoids have been shown to play a role in defence against $V$. longisporum [20]. An impact of development on resistance could be demonstrated. Systemic colonization of the upper parts of the plant starts with the onset of flowering in A. thaliana and Brassica spp. [8,9]. Direct or indirect signals that induce or promote flowering possibly affect the development of $V$. longisporum as well, for example, by stimulating the formation of mobile conidia or by directing growth of fungal hyphae. The loss of the vec 2 and vec3 QTL when using development as a covariate in QTL analyses indicates that the developmental differences on a physiological basis were still strong enough to cause the detection of these QTL, even though all plants had been assessed at the same phenotypic developmental stage. Developmental implications also played a role in stunting. The stunting resistance QTL on chromosome 4, r-stre3, 


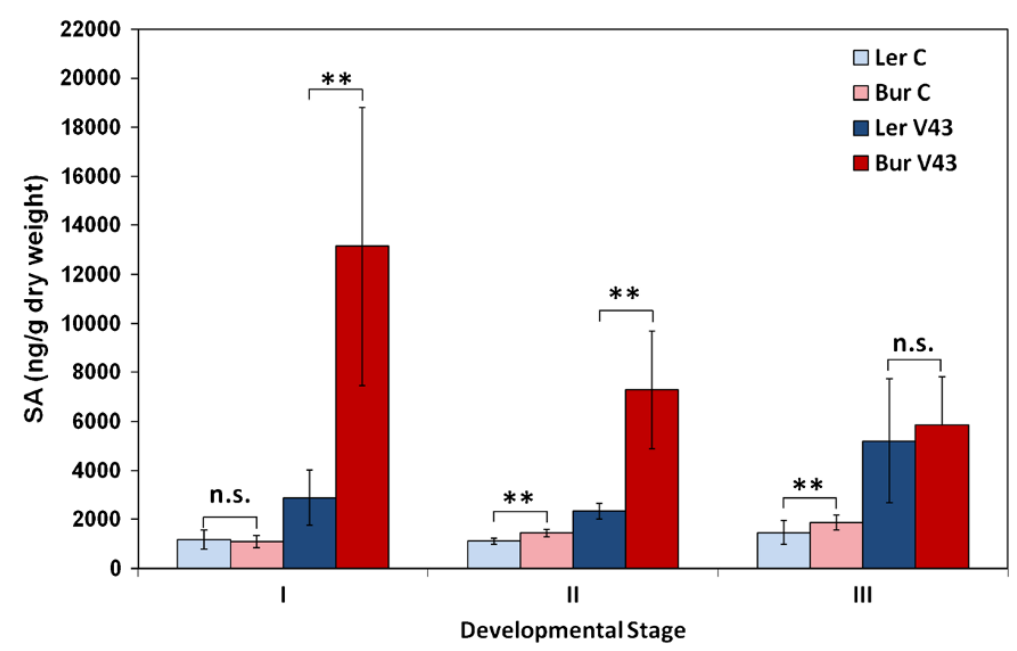

Figure 4 Contents of the phytohormone SA in Ler and Bur, mock-inoculated and inoculated, at different developmental stages. I: early-flowering stage, II: mid-flowering stage, III: beginning of silique maturity. $N=8$. Significance levels refer to differences of means between the ecotypes within one treatment (mock- and V. longisporum-inoculated, respectively) as determined by t-tests. Differences between mock- and $V$. longisporum-inoculated plants were always significant. V43 $=$ V. longisporum isolate 43.

similar to vec2 and vec3, disappeared when development was used as a covariate in MQM mapping.

The stunting resistance QTL on chromosomes 1 (stre1 and stre2) and 4 (r-stre3) depended on the season: They protected against stunting only during winter, which is consistent with prior observations for stre1 and stre2 [8]. Despite complementary lighting in the greenhouse, the development of $A$. thaliana was prolonged during winter (experiment 1) and developmental differences were more pronounced. Differences in light intensity and/or quality due to more natural illumination during summer are likely to cause these reactions. The strongest QTL on chromosome 1, stre2, co-localised with the Wakl9 gene, encoding a wall-associated kinase (WAK)-like protein [50]. A candidate for stre1 at the bottom of chromosome 1 is $R f o 1$ (Wakl22), encoding another WAK-like protein which has already been shown to play a role in $V$. longisporum resistance [16]. Since $V$. longisporum interacts with the host plant primarily in the apoplast [51], perception of pathogenesis-related molecules by cell-wall associated proteins may be crucial, and WAK proteins have been shown to mediate such perceptions [52]. Interestingly, several $\mathrm{Wak}$ genes have been shown to be induced by SA [53]. An SA induction of Wakl genes has not yet been shown, but it would correspond well with the early and strong SA induction detected in Bur. Increased SA levels have been observed to cause stunted growth also without disease [54]. The stunting effect of SA has been shown to be partially reverted by high-light conditions [55]. The combined effects of high levels of SA and lighting differences could explain the pronounced Verticillium-induced stunting mediated by the stre 1 and stre 2 alleles of Bur origin during winter. High levels of SA and its glucoside were also found in the xylem sap of $V$. longisporum-infected B. napus and were correlated to the degree of stunting and the amount of pathogen DNA [23]. Stunting is a common symptom in greenhouse or growth chamber experiments with $V$. longisporum occurring already at the rosette stage, but is never seen in infected field crops. Light intensity and quality is a major difference between field and greenhouse. This suggests that $V$. longisporum-induced stunting under experimental conditions also depends to a certain extent on the combination of high SA levels with artificial lighting.

The QTL controlling chlorosis seemed to be less affected by genes controlling development. The opposite has been reported in the literature [10]: A QTL delaying development and mediating resistance against Verticilliuminduced chlorosis, Vet1, has been identified in A. thaliana ecotype C24. Interestingly, the $r$-chl1 QTL on chromosome 4 co-localised with Vet1. The close proximity of several genes controlling the transition to flowering in this region (fri, cry1/hy, det1, ted1) complicates an interpretation of these results.

In the present study, Erecta has been shown to underlie the stunting resistance QTL $r$-stre1 on chromosome 2 , which explained a large part of the trait variation observed. Receptor-like kinases (RLKs) are often involved in controlling developmental processes or mediating disease resistance reactions [56]. The only Verticillium resistance gene identified so far, $V e 1$, also belongs to the RLK family $[57,58]$ and has been shown to recognize fungal effectors [59]. The leucine-rich repeat receptorlike Ser/Thr kinase ERECTA is an example of a signalling molecule controlling both developmental processes 

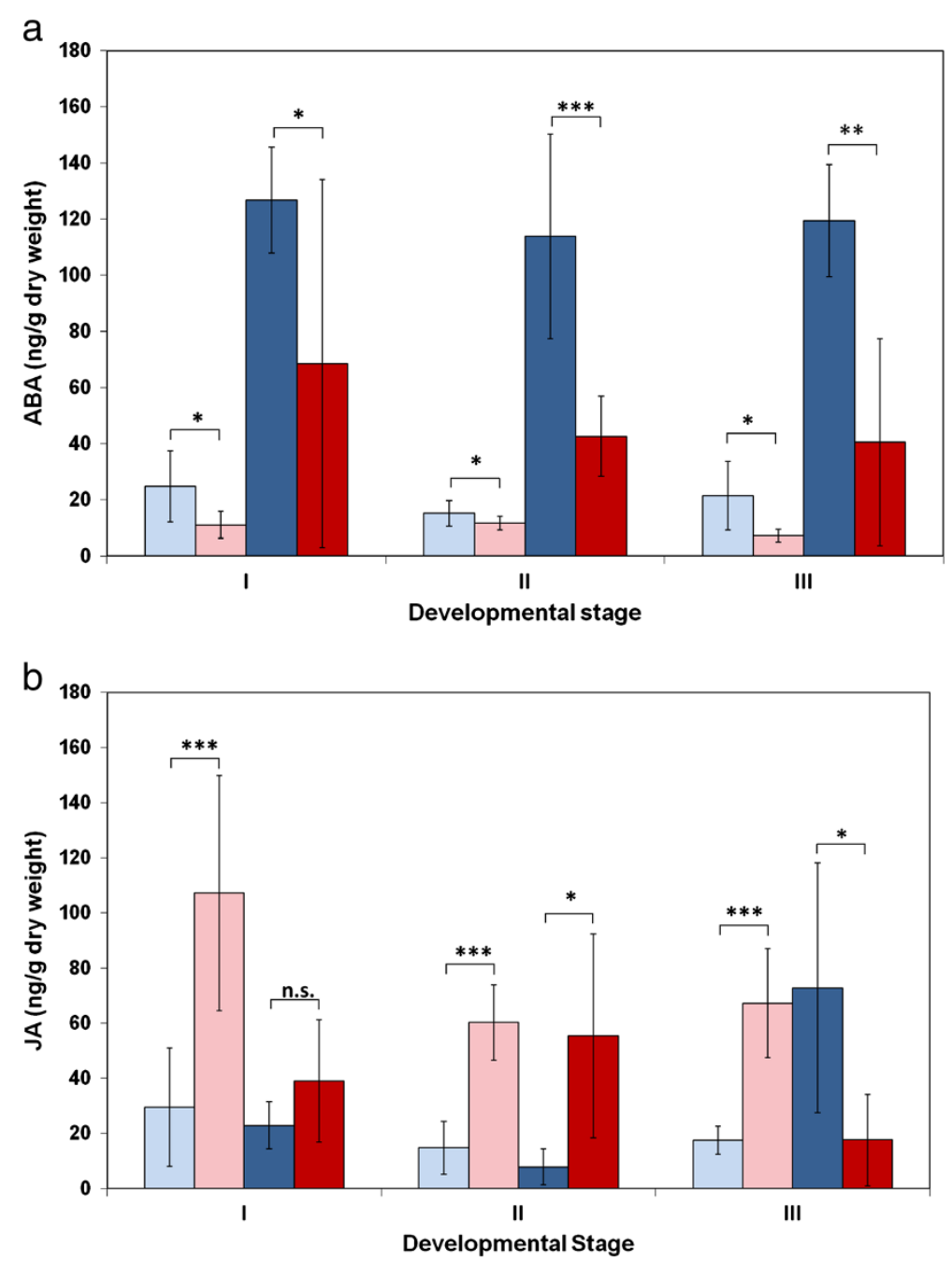

Figure 5 Contents of the phytohormones a) ABA and b) JA in Ler and Bur, mock-inoculated and inoculated, at different developmental stages. Light blue: Ler control, dark blue: Ler inoculated, pink: Bur control, red: Bur inoculated. I: early-flowering stage, II: mid-flowering stage, III: beginning of silique maturity. $N=8$. Significance levels refer to differences of means between the ecotypes within one treatment (mock-and $V$. longisporum-inoculated, respectively) as determined by t-tests. Differences between mock- and $V$. longisporum-inoculated plants were significant except for ABA in Bur at stage III, JA in Ler at stages I and II and JA in Bur at stage II ( $p<0.05, t$-test).

and QDR in A. thaliana [49]. erecta mutants show altered organ development resulting in compact growth $[32,60]$. Cell proliferation [61] and stomatal patterning [62] were also shown to be controlled by Erecta. Additionally, several resistance traits are reported to be controlled by Erecta in different pathosystems: Growth of the bacterial pathogen Ralstonia solanacearum was inhibited and wilt symptoms were reduced in A. thaliana plants with a functional Erecta gene compared to erecta mutants [63]. Infection with the necrotrophic fungus Plectosphaerella cucumerina resulted in more chlorosis and necrosis in erecta mutants compared to the respective WT accessions [64]. Enhanced susceptibility of erecta mutants, leading to enlarged leaf lesions, has also been reported for infection with the oomycete Pythium irregulare [65]. These results illustrate the close interconnection between ERECTA, development and QDR. Hence, it is not always clear whether altered disease resistance is a consequence of the developmental changes or a direct effect of ERECTA signalling. Studying other signalling components of the ERECTA pathway can shed light on this question. Plants defective in $A g b 1$ are morphologically distinct from erecta mutants [31], but defective in the same signalling pathway. In the present study, agb1-1 mutants were at least as susceptible to $V$. longisporum-induced stunting as the erecta mutants. This supports the view that a functional ERECTA pathway mitigates the stunting effect of $V$. longisporum infection independently of its effect on morphology. 

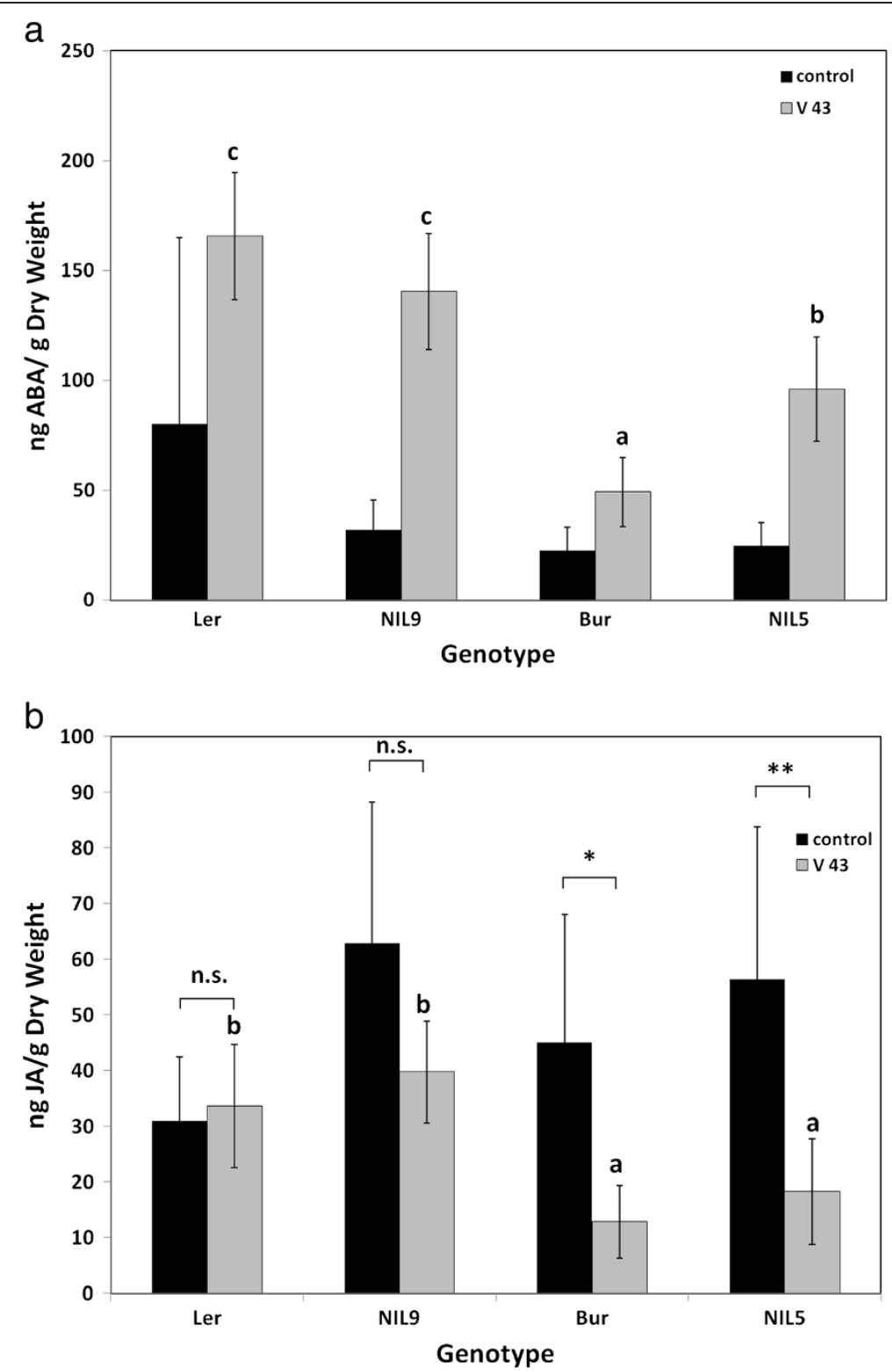

Figure 6 Contents of the phytohormones a) ABA and b) JA in Bur, Ler and two near-isogenic lines. NIL9 contained Ler alleles in the variable region and NIL5 contained Bur alleles. a): Differences between mock- and V. longisporum-inoculated plants were significant ( $n=6-7$, $p<0.05)$. b) Asterisks refer to the significance level of differences between mock-inoculated and $V$. longisporum-treated plants within one genotype ( $t$-test, $n=6$ ). Mean contents of inoculated plants marked with different letters differed significantly at $p<0.05$. Mean phytohormone contents of controls did not differ significantly (one-way ANOVA and post-hoc Tukey test). Vertical bars denote standard deviations. $V 43=V$. longisporum isolate 43.

Resistance to systemic colonization was not enhanced by a functional Erecta gene in La-0 compared to Ler. This is strong evidence that Erecta, although it is located in vec1, is not involved in mediating resistance to systemic colonization by $V$. longisporum. A tendency towards stronger colonization of erecta mutants in the moderately susceptible Col-0 background compared to WT was never significant, and is interpreted as an indirect effect of the reduced plant height caused by erecta.
$V$. longisporum may reach the apex of a shorter shoot more easily. This view is corroborated by the fact that the long stalks of the agb1-1 mutant were very poorly colonized, thus behaving very differently from the erecta mutants, despite the fact that both AGB1 and ERECTA act in the same signalling pathway.

Altogether, Erecta has been proven to be a source of natural genetic variation in quantitative resistance not only to $V$. longisporum. Not much is known so far about 
the allelic variation of Erecta beyond the level of complete loss of function and its consequences for disease resistance in natural accessions. A comprehensive analysis of Erecta, its structural and regulatory variability and its homologues in cruciferous crop plants would, therefore, be desirable.

$\mathrm{ABA}$ and JA contents differed in a genotype-specific way that correlated with the rates of fungal colonization. It is not known whether $V$. longisporum produces ABA itself, which could explain the high levels in heavily colonized plants. In $B$. napus, however, $V$. longisporum infection did not increase ABA levels in xylem sap [23]. The sampling was not fully comparable in both studies, as different parts of the stalk were sampled and the samples in B. napus were taken at an earlier developmental stage. The results in $B$. napus make it more likely that different ABA levels depended on the host genotypes instead of fungal biomass.

The role of ABA signalling in $V$. longisporum resistance is complex. The ABA-deficient aba2-1 mutant was highly susceptible to $V$. longisporum-induced stunting, but other ABA signalling mutants were unaffected, indicating that a specific function of ABA2 was involved in the response observed that did not require $A B A$ in general [16]. From the many functions of ABA in development and disease, different effects can be assumed in the context of Verticillium disease. ABA has been shown to influence plant defence reactions in various ways [66]. In most cases, ABA increased susceptibility to pathogens due to suppression of SA synthesis [67] and/or antagonism with jasmonate-ethylene signalling [68]. However, ABA can also stimulate JA biosynthesis and increase resistance [65]. ABA has important functions during seed development, such as a trigger for the acquisition of storage molecules during cell enlargement [69]. ABA plays a significant role in the induction of senescence [70]. A major effect of $V$. longisporum on B. napus in the field is the induction of premature ripening, which can be recognized by chlorotic stems and which is leading to reduced seed size. Increased levels of ABA might support the deviation of mobilised nutrients to foster the growth of fungal biomass. Furthermore, it is possible that clogged vessels after $V$. longisporum-infection induce increased ABA levels as a result of drought stress. However, it has recently been shown that $V$. longisporum infection can even increase drought tolerance of the host as a consequence of de-novo xylem formation [29]. Studying xylem trans-differentiation in susceptible and resistant genotypes could clarify the relevance of xylem formation for resistance.

JA contents decreased after infection with $V$. longisporum in Bur and the NIL that contained Bur alleles in the region of vec1. Plant-pathogenic Verticillium species are regarded as hemibiotrophs with a necrotrophic phase during the late stages of infection. Defence responses against necrotrophic pathogens are often induced by JA [71]. Accordingly, JA-deficient tomato plants have been shown to be more susceptible to Verticillium dahliae than WT plants [72]. In the present study, however, the colonization-resistant ecotype Bur was characterized by a decrease in JA levels after infection. Since JA is also involved in A. thaliana leaf senescence [73], $V$. longisporum possibly benefits from senescence processes induced by JA and ABA, which would suggest a stimulation of hormone production by the fungus. Alterations of senescence-like processes were also postulated to underlie increased resistance of the A. thaliana JA-receptor mutant coil against $V$. longisporum colonization [24]; however, this disease-promoting effect of Coil has been shown to be JA-independent. These results confirm that fine-tuning of a resistance reaction by cross-talk of phytohormone signalling pathways is highly individual for each pathosystem [74]. In addition, possible manipulations of the host's hormone status by the pathogen for its own benefit should be considered.

\section{Conclusions}

Phytohormone signalling processes have been demonstrated to be subject to allelic variation and underlie QDR against $V$. longisporum in natural $A$. thaliana accessions. ERECTA, SA, ABA, and JA signalling has been shown to mediate an ecotype-specific response of $A$. thaliana to $V$. longisporum infection. The ecotypespecific differences for $\mathrm{ABA}$ and JA contents were mediated by the same genomic region on chromosome 2 that also controlled systemic colonization by $V$. longisporum. This region contains the major QTL controlling systemic colonization, vec1, and also Erecta. As colonization resistance was shown to be independent of Erecta, this region should contain other gene(s) that control the degree of fungal colonization. This type of resistance is likely to depend on ABA and JA signalling, as both NILs differed for these hormone contents in the same way as the parental lines that contributed the respective alleles in the polymorphic region. Ongoing studies on differential gene expression depending on vec1 should allow one to draw a more comprehensive picture of the defence reactions leading to colonization resistance. Furthermore, cloning of a gene that controls this reaction should be feasible by combining map-based confinement of the QTL region with expression analysis. It was demonstrated that different resistance traits were controlled independently on a physiological basis, but were still genetically linked. Understanding the biological basis of phenotypic variation in $A$. thaliana with respect to $V$. longisporum resistance will provide new approaches for implementing durable resistance in cruciferous crops. 


\section{Additional files}

Additional file 1: Marker information. Lists all markers that have been used for genotyping the (BurXLer) RIL population. It consists of two tables. Table $\mathbf{S} \mathbf{1}$ is an overview of all markers used, their type, chromosomal location, and their source. In Table S2, the technical information of all new markers is listed. This includes primer information, melting temperature of primers, information on the polymorphism, and the PCR protocol.

Additional file 2: Genetic and physical map. Shows the genetic map and the physical map of the (BurXLer) RIL population. The genetic map shows marker distances for all chromosomes in $\mathrm{cM}$, the physical map shows the location of markers in kilobases (kb) according to the AGI map [48].

Additional file 3: QTL information. Lists information for all QTL detected in the present study with 94 (BurXLer) RILs in two separate experiments. QTL for the traits systemic colonization, stunting resistance and resistance to chlorosis detected with different parameters are listed in a table. The chromosomal location, the LRS peak position(s), the LOD score, the additive component, the explained trait variance, and allelic means are given for each QTL.

Additional file 4: Frequency distribution of phenotypic data. Shows the frequency distribution histograms for the phenotypic data used in QTL mapping. Histograms are shown for the parameters "\% colonized shoot segments", "pg Verticillium DNA/mg fresh weight", "performance height", "Mean number of yellow leaves in V. longisporum-inoculated plants", "Mean number of yellow leaves related to total rosette leaf number", and "Mean difference in yellow leaves between inoculated and mock-inoculated plants".

Additional file 5: Systemic colonization of Erecta signalling mutants and corresponding WT-lines. Contains a bar chart visualizing systemic colonization of erecta mutants, agb1-1 mutant and the corresponding WT genotypes. It provides evidence that Erecta is not involved in mediating resistance to systemic colonization by $V$. longisporum.

Additional file 6: SA contents in Bur, Ler and NILs. Contains a bar chart visualizing SA contents in Bur, Ler and two NILs differing in the vec1 region. The data provide evidence that SA hyperinduction, which is characteristic for Bur, is not associated with the vec1 locus.

Additional file 7: Phytohormone contents of erecta mutants, corresponding WT-lines and Bur. Contains three bar charts visualizing $\mathrm{SA}, \mathrm{ABA}$ and JA contents of Erecta signalling mutants and corresponding WT lines after mock-inoculation and $V$. longisporum-infection. Additional file 7 provides evidence that differences in phytohormone response to $\mathrm{V}$. longisporum between Bur and Ler are not caused by Erecta.

\section{Competing interests}

The authors declare that there are no competing interests.

\section{Authors' contributions}

EH planned all the plant experiments, supervised experiment 3, carried out all other plant experiments, analysed the data, and drafted the manuscript. PK provided the GPCR data on fungal biomass and helped to draft the manuscript. RS provided all data on phytohormone contents and helped to draft the manuscript. AT carried out experiment 3, collected the data and helped with their analysis. ED conceived of the study, participated in its design and coordination, participated in QTL analysis, and helped to draft the manuscript. All authors read and approved the final manuscript.

\section{Acknowledgements}

We gratefully acknowledge financial support from Deutsche Forschungsgemeinschaft (DFG), Bonn, Germany (Di1501/1-1, Di 1502/3-1 and KA 1209/8-1 grants) and Norddeutsche Pflanzenzucht (NPZ), Germany. We are grateful to Thomas Schmülling for hosting and supporting this project and we thank Claudia Delbrügge, Katharina Fraunhofer, Anika Krüger, Christiane Manthey, and Heike Rollwage for excellent technical support. Karen Zeise, Berlin, Germany, is gratefully acknowledged for supplying the fungal strain.

\section{Author details}

${ }^{1}$ Freie Universität Berlin, Fachbereich Biologie, Chemie, Pharmazie, Institut für Biologie, Dahlem Centre of Plant Sciences, Angewandte Genetik, Albrecht-Thaer-Weg 6, 14195 Berlin, Germany. ${ }^{2}$ Department of Crop Sciences, Georg-August-Universität Göttingen, Molecular Phytopathology and Mycotoxin Research Section, Grisebachstraße 6, 37077 Göttingen, Germany.

Received: 25 October 2013 Accepted: 13 March 2014

Published: 1 April 2014

\section{References}

1. Poland JA, Balint-Kurti PJ, Wisser RJ, Pratt RC, Nelson RJ: Shades of gray: the world of quantitative disease resistance. Trends Plant Sci 2008, 14:21-29.

2. St.Clair D: Quantitative disease resistance and quantitative resistance loci in breeding. Annu Rev Phytopathol 2010, 48:247-268.

3. Inderbitzin P, Davis R, Bostock R, Subbarao K: The ascomycete Verticillium longisporum is a hybrid and a plant pathogen with expanded host range. PLoS One 2011, 6:e18260.

4. Dixelius C, Happstadius I, Berg G: Verticillium wilt on Brassica oil crops - a Swedish perspective. J Swed Seed Assoc 2005, 115:36-48.

5. Dunker S, Keunecke H, Steinbach P, von Tiedemann A: Impact of Verticillium longisporum on yield and morphology of winter oilseed rape (Brassica napus) in relation to systemic spread in the plant. J Phytopathol 2008, 156:698-707.

6. Gladders P: Relevance of Verticillium wilt (Verticillium longisporum) in winter oilseed rape in the UK. HCGA Research Rev 2009, 72:1-39.

7. Krüger W: Untersuchungen zur Verbreitung von Verticillium dahliae Kleb. und anderen Krankheits- und Schaderregern bei Raps in der Bundesrepublik Deutschland. Nachrichtenb/ Deutsch Pflanzenschutzd 1989, 41:49-56.

8. Häffner E, Karlovsky P, Diederichsen E: Genetic and environmental control of the Verticillium syndrome in Arabidopsis thaliana. BMC Plant Biol 2010, $10: 235$.

9. Zhou L, Hu Q, Johansson A, Dixelius C: Verticillium longisporum and V. dahliae: infection and disease in Brassica napus. Plant Pathol 2006, 55:137-144.

10. Veronese P, Narasimhan ML, Stevenson RA, Zhu J-K, Weller SC, Subbarao KV, Bressan RA: Identification of a locus controlling Verticillium disease symptom response in Arabidopsis thaliana. Plant J 2003, 35:574-587.

11. Johansson A, Goud J-KC, Dixelius C: Plant host range of Verticillium longisporum and microsclerotia density in Swedish soils. Eur J Plant Pathol 2006, 114:139-149.

12. Debode J, Declercq B, Höfte M: Identification of cauliflower cultivars that differ in susceptibility to Verticillium longisporum using different inoculation methods. J Phytopathol 2005, 153:257-263.

13. Happstadius I, Ljungberg A, Kristiansson B, Dixelius C: Identification of Brassica oleracea germplasm with improved resistance to Verticillium wilt. Plant Breed 2003, 122:30-34.

14. Rygulla W, Snowdon RJ, Eynck C, Koopmann B, Tiedemann A, Lühs W, Friedt W: Broadening the genetic basis of Verticillium longisporum resistance in Brassica napus by interspecific hybridisation. Phytopathology 2007, 97:1391-1396.

15. Rygulla W, Snowdon RJ, Friedt W, Happstadius I, Cheung WY, Chen D: Identification of quantitative trait loci for resistance against Verticillium longisporum in oilseed rape (Brassica napus). Phytopathology 2008, 98:215-221.

16. Johansson A, Staal J, Dixelius C: Early responses in the Arabidopsis-Verticillium longisporum pathosystem are dependent on NDR1, JA- and ET-associated signals via cytosolic NPR1 and RFO1. Mol Plant Microbe Interact 2006, 19:958-969.

17. Steventon LA, Okori P, Dixelius C: An investigation of the susceptibility of Arabidopsis thaliana to isolates of two species of Verticillium. $J$ Phytopathol 2001, 149:395-401.

18. Eynck C, Koopmann B, Karlovsky P, von Tiedemann A: Internal resistance in winter oilseed rape inhibits systemic spread of the vascular pathogen Verticillium longisporum. Phytopathology 2009, 99:802-811.

19. Obermeier C, Hossain M, Snowdon R, Knüfer J, von Tiedemann A, Friedt W: Genetic analysis of phenylpropanoid metabolites associated with resistance against Verticillium longisporum in Brassica napus. Mol Breed 2013, 31:347-361.

20. König S, Feussner K, Kaever A, Landesfeind M, Thurow C, Karlovsky P, Gatz C, Polle A, Feussner I: Soluble phenylpropanoids are involved in the defense 
response of Arabidopsis against Verticillium longisporum. New Phytol 2014. http://onlinelibrary.wiley.com/doi/10.1111/nph.12709/full.

21. Diener AC, Ausubel FM: Resistance to fusarium oxysporum 1, a dominant Arabidopsis disease-resistance gene, is not race-specific. Genetics 2005, 171:305-321.

22. Pantelides IS, Tjamos SE, Paplomatas EJ: Ethylene perception via ETR1 is required in Arabidopsis infection by Verticillium dahliae. Mol Plant Pathol 2010, 11:191-202.

23. Ratzinger A, Riediger N, von Tiedemann A, Karlovsky P: Salicylic acid and salicylic acid glucoside in xylem sap of Brassica napus infected with Verticillium longisporum. J Plant Res 2009, 122:571-579.

24. Ralhan A, Schöttle S, Thurow C, Iven T, Feussner I, Polle A, Gatz C: The vascular pathogen Verticillium longisporum requires a jasmonic acid-independent COI1 function in roots to elicit disease symptoms in Arabidopsis shoots. Plant Physiol 2012, 159:1192-1203.

25. Iven T, König S, Singh S, Braus-Stromeyer SA, Bischoff M, Tietze LF, Braus G, Lipka V, Feussner I, Dröge-Laser W: Transcriptional activation and production of tryptophan-derived secondary metabolites in Arabidopsis roots contributes to the defense against the fungal vascular pathogen Verticillium longisporum. Mol Plant 2012, 5:1389-1402.

26. Singh S, Braus-Stromeyer S, Timpner C, Valerius O, von Tiedemann A Karlovsky P, Druebert C, Polle A, Braus G: The plant host Brassica napus induces in the pathogen Verticillium longisporum the expression of functional catalase peroxidase which is required for the late phase of disease. Mol Plant Microbe Interact 2012, 25:569-581.

27. Yadeta $K$, Hanemian M, Smit P, Hiemstra J, Pereira A, Marco Y, Thomma BP: The Arabidopsis thaliana DNA-binding protein AHL19 mediates Verticillium wilt resistance. Mol Plant Microbe Interact 2011, 24:1582-1591.

28. Floerl S, Druebert C, Majcherczyk A, Karlovsky P, Kües U, Polle A: Defense reactions in the apoplastic proteome of oilseed rape (Brassica napus var. napus) attenuate Verticillium longisporum growth but not disease symptoms. BMC Plant Biol 2008, 8:129.

29. Reusche M, Thole K, Janz D, Truskina J, Rindfleisch S, Drübert C, Polle A, Lipka $V$, Teichmann T: Verticillium infection triggers VASCULAR-RELATED NAC DOMAIN7-dependent de novo xylem formation and enhances drought tolerance in Arabidopsis. Plant Cell 2012, 24:3823-3837.

30. Kranz AR, Kirchheim B: Genetic resources in Arabidopsis. Arabidopsis Inform Serv Newslett 1985, 24:1-368.

31. Lease KA, Wen J, Li J, Doke JT, Liscurn E, Walker JC: A mutant Arabidopsis heteromeric $\mathrm{G}$-protein $\beta$ subunit affects leaf, flower, and fruit development. Plant Cell 2001, 13:2631-2641.

32. Torii KU, Mitsukawa N, Oosumi T, Matsuura Y, Yokoyama R, Whittier RF, Komeda Y: The Arabidopsis ERECTA gene encodes a putative receptor protein kinase with extracellular leucine-rich repeats. Plant Cell 1996, 8:735-746.

33. Zeise $K$, von Tiedemann A: Host specialization among vegetative compatibility groups of Verticillium dahliae in relation to Verticillium longisporum. J Phytopathol 2002, 150:112-119.

34. The Arabidopsis information resource (TAIR). [http://www.arabidopsis.org]

35. O'Neill CM, Morgan C, Kirby J, Tschoep H, Deng PX, Brennan M, Rosas U, Fraser F, Hall C, Gill S, Bancroft I: Six new recombinant inbred populations for the study of quantitative traits in Arabidopsis thaliana. Theor Appl Genet 2008, 116:623-634.

36. Warthmann N, Fitz J, Weigel D: MSQT for choosing SNP assays from multiple DNA alignments. Bioinformatics 2007, 23:2784-2787.

37. Nordborg M, Hu TT, Ishino Y, Jhaveri J, Toomajian C, Zheng H, Bakker E, Calabrese P, Gladstone J, Goyal R, Jakobsson M, Kim S, Morozov Y Padhukasahasram B, Plagnol V, Rosenberg NA, Shah C, Wall JD, Wang J, Zhao K, Kalbfleisch T, Schulz V, Kreitman M, Bergelson J: The pattern of polymorphism in Arabidopsis thaliana. PLoS Biol 2005, 3:1289-1299.

38. Ossowski S, Schneeberger K, Clark RM, Lanz C, Warthmann N, Weigel D: Sequencing natural strains of Arabidopsis thaliana with short reads. Genome Res 2008, 18:2024-2033.

39. Webcutter 2.0. [http://rna.lundberg.gu.se/cutter2/]

40. Brandfass C, Karlovsky P: Upscaled CTAB-based DNA extraction and real-time $P C R$ assays for Fusarium culmorum and $F$. graminearum DNA in plant material with reduced sampling error. Int J Mol Sci 2008, 9:2306-2321.

41. Splivallo R, Fischer U, Gobel C, Feussner I, Karlovsky P: Truffles regulate plant root morphogenesis via the production of auxin and ethylene. Plant Physiol 2009, 150:2018-2029.
42. van Ooijen J, Voorrips R: Joinmap Version 4.0: Software for the Calculation of Genetic Linkage Maps. Wageningen, The Netherlands: Plant Research International; 2008

43. Manly KF, Cudmore RH, Meer JM: Map Manager QTX, cross-platform software for genetic mapping. Mamm Genome 2001, 12:930-932

44. Collard BCY, Jahufer MZZ, Brouwer JB, Pang ECK: An introduction to markers, quantitative trait loci (QTL) mapping and marker-assisted selection for crop improvement: The basic concepts. Euphytica 2005, 142:169-196.

45. van Ooijen J: MapQTL R 6, Software for the mapping of quantitative trait loci in experimental populations of diploid species. Edited by Kyazma BV. The Netherlands: Wageningen; 2009.

46. Voorrips RE: Mapchart 2.2. [http://www.wageningenur.nl/en/show/Mapchart.htm]

47. IBM corp: SPSS Statistics 20. Armonk NY: IBM corp; 2011.

48. The Arabidopsis Genome Initiative (AGI): Analysis of the genome sequence of the flowering plant Arabidopsis thaliana. Nature 2000, 408:796-815.

49. van Zanten M, Snoek LB, Proveniers MCG, Peeters AJM: The many functions of ERECTA. Trends in Plant Sci 2009, 14:214-218.

50. Verica JA, He Z-H: The cell wall-associated kinase (WAK) and WAK-like Kinase gene family. Plant Physiol 2002, 129:455-459.

51. Floerl S, Majcherczyk A, Possienke M, Feussner K, Tappe H, Gatz C, Feussner I, Kües $U$, Polle A: Verticillium longisporum affects the leaf apoplastic proteome, metabolome, and cell wall properties in Arabidopsis thaliana. PLoS One 2012, 7:e31435

52. Brutus A, Sicilia F, Macone A, Cervone F, DeLorenzo G: A domain swap approach reveals a role of the plant wall-associated kinase 1 (WAK1) as a receptor of oligogalacturonides. Proc Natl Acad Sci 2010, 107:9452-9457.

53. He Z-H, Cheeseman I, He D, Kohorn BD: A cluster of five wall-associated receptor kinase genes, Wak1-5, are expressed in specific organs of Arabidopsis. Plant Mol Biol 1999, 39:1189-1196.

54. Rivas-San-Vicente M, Plasencia J: Salicylic acid beyond defense: its role in plant growth and development. Exp Bot 2011, 62:3321-3338.

55. Mateo A, Funck D, Mühlenbock P, Kular B, Mullineaux PM, Karpinski S: Controlled levels of salicylic acid are required for optimal photosynthesis and redox homeostasis. J Exp Bot 2006, 57:1795-1807.

56. Shiu SH, Bleecker AB: Plant receptor-like kinase gene family: diversity, function and signaling. SCi STKE 2001, 113:re22.

57. Fradin EF, Zhang Z, Ayala JCJ, Castroverde CDM, Nazar RN, Robb J, Liu C-M, Thomma BP: Genetic dissection of Verticillium wilt resistance mediated by tomato Ve1. Plant Physiol 2009, 150:320-332.

58. Kawchuk LM, Hachey J, Lynch DR, Kuksar F, van Rooijen G, Waterer DR, Robertson A, Kokko E, Byers R, Howard RJ, Fischer R, Prüfer J: Tomato Ve disease resistance genes encode cell surface-like receptors. Proc Natl Acad Sci 2001, 98:6511-6515.

59. de Jonge R, van Esse HP, Maruthachalam K, Bolton MD, Santhanam P, Saber MK, Zhang Z, Usami T, Lievens B, Subbarao KV, Thomma BP: Tomato immune receptor Ve1 recognises effector of multiple fungal pathogens uncovered by genome and RNA sequencing. Proc Natl Acad Sci 2012, 109:5110-5115.

60. Rédei G: Supervital mutants of Arabidopsis. Genetics 1962, 47:443-460.

61. Shpak ED, Berthiaume CT, Hill EJ, Torii KU: Synergistic interaction of three ERECTA-family receptor-like kinases controls Arabidopsis organ growth and flower development by promoting cell proliferation. Development 2004, 131:1491-1501.

62. Shpak ED, McAbee JM, Pillitteri LJ, Torii KU: Stomatal patterning and differentiation by synergistic interactions of receptor kinases. Science 2005, 309:290-293.

63. Godiard L, Sauviac L, Torii KU, Grenon O, Mangin B, Grimsley NH, Marco Y: ERECTA, an LRR receptor-like kinase protein controlling development pleiotropically affects resistance to bacterial wilt. Plant J 2003, 36:353-365.

64. Llorente F, Alonso-Blanco C, Sánchez-Rodriguez C, Jorda L, Molina A ERECTA receptor-like kinase and heteromeric $\mathrm{G}$ protein from Arabidopsis are required for resistance to the necrotrophic fungus Plectosphaerella cucumerina. Plant J 2005, 43:165-180.

65. Adie B, Pérez-Pérez J, Pérez-Pérez M, Godoy M, Sánchez-Serrano J-J, Schmelz $E$, Solano $R: A B A$ is an essential signal for plant resistance to pathogens affecting JA biosynthesis and the activation of defenses in Arabidopsis. Plant Cell 2007, 19:1665-1681.

66. Asselbergh B, de Vleesschauer D, Höfte M: Global switches and fine tuning ABA modulates plant pathogen defense. Mol Plant Microbe Interact 2008, 21:709-719. 
67. Audenaert K, de Meyer GB, Höfte M: Abscisic acid determines basal susceptibility of tomato to Botrytis cinerea and suppresses salicylic acid-dependent signaling mechanisms. Plant Physiol 2002, 128:491-501.

68. Anderson JP, Badruzsaufari E, Schenk PM, Manners JM, Desmond OJ, Ehlert C, Maclean DJ, Ebert PR, Kazan K: Antagonistic interaction between abscisic acid and jasmonate-ethylene signaling pathways modulates defense gene expression and disease resistance in Arabidopsis. Plant Cell 2004, 16:3460-3479.

69. Finkelstein RR, Gibson Sl: ABA and sugar interactions regulating development: cross-talk or voices in a crowd? Curr Opin Plant Biol 2001, 5:26-32.

70. Schippers JHM, Jing HC, Hille J, Dijkwel PP: Developmental and hormonal control of leaf senescence. In Senescence Processes in Plants. Edited by Gan S. Oxford UK: Blackwell Publishing; 2007:145-170.

71. Glazebrook J: Contrasting mechanisms of defense against biotrophic and necrotrophic pathogens. Annu Rev Phytopathol 2005, 43:205-227.

72. Thaler JS, Owen B, Higgins VJ: The role of jasmonate response in plant susceptibility to diverse pathogens with a range of lifestyles. Plant Physio 2004, 135:530-538.

73. He Y, Fukushige H, Hildebrand DF, Gan S: Evidence supporting a role of jasmonic acid in Arabidopsis leaf senescence. Plant Physiol 2002, 128:876-884.

74. Pieterse CMJ, Leon-Reyes A, van der Ent S, van Wees SCM: Networking by small-molecule hormones in plant immunity. Nat Chem Biol 2009, 5:308-316.

doi:10.1186/1471-2229-14-85

Cite this article as: Häffner et al.: ERECTA, salicylic acid, abscisic acid, and jasmonic acid modulate quantitative disease resistance of Arabidopsis thaliana to Verticillium longisporum. BMC Plant Biology 2014 14:85.

\section{Submit your next manuscript to BioMed Central and take full advantage of:}

- Convenient online submission

- Thorough peer review

- No space constraints or color figure charges

- Immediate publication on acceptance

- Inclusion in PubMed, CAS, Scopus and Google Scholar

- Research which is freely available for redistribution 